\title{
EXCESS OF SOCIAL ACTIVITY REDUCES THE RESPONSIVENESS OF SWARMS
}

\author{
DAVID MATEO \& ROLAND BOUFFANAIS \\ Singapore University of Technology and Design, Somapah Road, Singapore, Singapore.
}

\begin{abstract}
Social interaction increases significantly the collective action and operation of swarming systems. However, a growing body of empirical results reveals limitations in the number of social links in natural swarms, thereby suggesting possibly detrimental effects associated with excessive social activity. We study the consequences that such excess has on the responsiveness of a swarm facing dynamic perturbations. Using a classical model of collective motion, we show that the capacity of a swarm to perform actions critical to its survival peaks for a number of social links much smaller than the maximum possible number. This relationship between social activity, responsiveness and predator avoidance capacity presents a plausible picture of how natural selection could favor organisms that self-limit their sociality, as is common in many taxa. These results have important implications for the design of swarm robotics systems.
\end{abstract}

Keywords: collective behaviors, consensus dynamics, sociality, swarming systems.

\section{INTRODUCTION}

Animal groups provide paradigmatic examples of collective phenomena in which repeated interactions among individuals produce dynamic patterns and responses on a scale larger than individuals themselves. Some of the examples around us include the coordinated movements of fish and birds in a school or a flock respectively, the chemotactic aggregation of amoebae, the formation of lanes in densely-packed human crowds, the generation of vortices in bacterial colonies, the synchronized march of wingless locusts and the synchronized flashing of fireflies. Many more examples can also be found inside all of us: the firing of neurons in our brains, the clustering of differentiated cells to construct our organs both during embryonic development and wound healing, and the targeted response of neutrophils as part of the initial immune response to a bacterial infection. This nonexhaustive list of collective behaviors of unicellular and multicellular organisms is revealing of the pervasiveness of swarming in the natural world [1].

Swarming agents are interconnected organisms or agents. A key benefit of being connected is access to information. Such dynamic interconnectivity serves the purpose of channeling information exchanges, which are critical to the effectiveness in swarming. Indeed, it is well known that collective animal behavior is dependent on the existence of communication channels enabling information exchange between individuals [1,2]. For instance, the collective surveillance against oncoming threats of a flock of birds provides a higher level of vigilance only if the information obtained by each pair of eyes is shared among the flock.

Swarm dynamics is governed by inter-agent interactions and also interactions between individual agents and their environment. Through all these interactions a social transmission of information occurs, eventually leading to a wide range of self-organizing natural behaviors. Let us come back to the example of the evasive maneuver performed by a flock of birds confronting a threat. The predator 
is external to the flock and can be said to be part of the environment. The detection of its presence by birds at a close range amounts to an external signal or stimuli - a.k.a. sensory information. The detecting agents swerve to move away from this stimuli, thereby creating a signal internal to the flock - behavioral information. This behavioral signal contains information and propagates through the flock at high speed and with little distortion [3, 4]. This latter information transmission process can be said to be responsible for the overall effectiveness of the flock in dodging the predator's attack. For instance, birds detecting an environmental threat give utmost priority to sensory information, thereby discarding behavioral signals from neighboring conspecifics. In short, information is a crucial currency for animals from a behavioral standpoint. This is certainly also true for artificial swarms.

Tracing the flow of information and quantifying informational exchanges are keys to gaining insight into the functioning of swarms. It also plays a central role when designing swarming systems. From the engineering standpoint, such effective information transfers highlight the existence of an underlying communication channel that takes the form of the swarm signaling network (SSN) [5] as is schematically shown in Fig. 1. Animal collectives use this SSN to effectively respond to changes in the surroundings: e.g. coordinated evasive maneuvers upon detection of a predator or collision avoidance. The structure and dynamics of SSNs are quite unique, being temporal and adaptive networks [6] with a dynamics deeply interwoven with the agents' motion dynamics embedded in the physical space. For instance, in the particular case of bird flocks governed by a topological interaction [7], the SSN has been found to be a small-world, homogeneous clustered network whose connectedness is key to yielding resilient swarming behaviors [5]. The knowledge of and access to the structural properties of the SSN revealed the high dynamic controllability of swarms [5, 8] where few agents are capable of driving the dynamics of the swarm as a whole - as well as very effective consensus reaching processes [9].

Increasing the amount of social links is usually known to yield an improved collective operation. From a network-theoretic standpoint, this amounts to increasing the degree distribution of the signaling network. However, there is a growing body of evidence showing that most collective behaviors

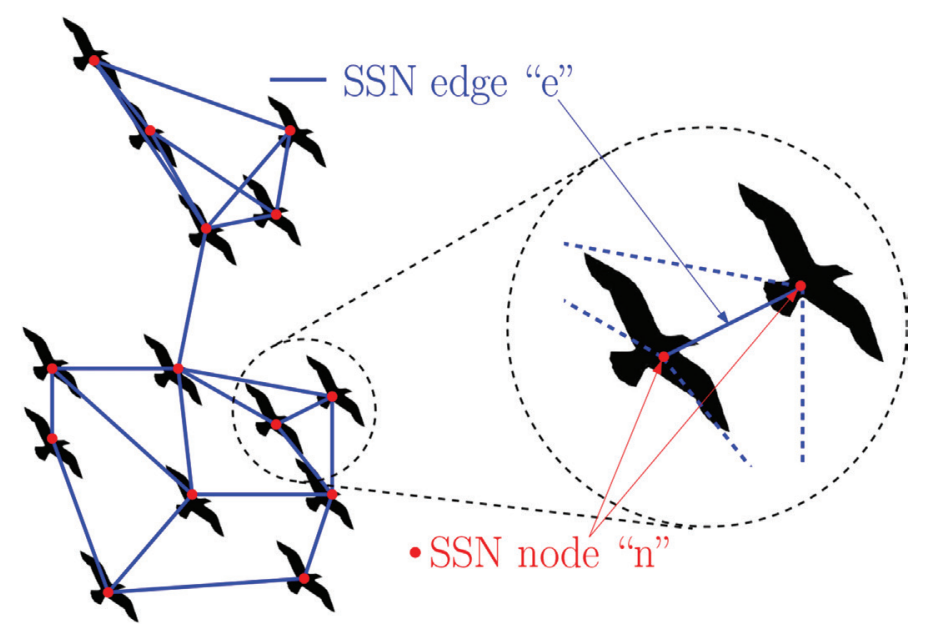

Figure 1: Schematic of a networked flock of birds with the associated information transmission channel in the form of the SSN. Edges represent an interaction between two agents. Nodes are the agents themselves, which act as routers for the behavioral information. 
naturally occurring involve a limited number of social links - i.e. the SSN has a very specific degree distribution. A recent landmark study from the STARFLAG group imaged and tracked wild flocks of starlings numbering in the thousands and revealed that the neighborhood of interaction actually depends on a topological distance [7, 10]. More precisely, Ballerini et al. [7] discovered that each bird interacts on average with a fixed number of nearest neighbors (six to eight). This apparent limitation in the number of social links in flocks of starlings was further observed with swarms of midges [11] governed by a metric interaction distance and in which a regulation of the nearest neighbor distance, as a function of the size of the swarm, takes place. Similar limitations in the number of social links have been observed with other taxa, including insects: e.g. the ants (Lasius fuliginosus ) regulate their rate of social encounters [12]. The important fact here is that this limitation in the number of social links established appears to be a behavioral feature and not a direct result of physical limitations associated with their sensing and/or signaling apparatuses. These findings suggest that an excess in social connectivity could have detrimental consequences for the swarm dynamics, when the latter is confronted with changing circumstances.

Here, we investigate the consequences of establishing an excessive amount of social links on the collective dynamics of a swarming system confronted with a threat. Understanding these effects has far-reaching consequences on the design of artificial swarms [13] and for the study of spreading of behaviors in online communities [14]. Previous studies of this problem [9, 15] were focused on system reaching consensus and have shown that, above a certain point, increasing the number of social links yields only marginal benefit to the collective. However, a systematic study of the responsiveness of swarms as a function of the number of social connections is lacking. To this aim, we use a canonical model of collective motion, in which agents move by adjusting their direction of travel to that of their neighbors in both two and three-dimensional setups. We characterize the responsiveness of the swarm by simulating a predator attack and measuring the survival rate of agents depending on their level of social activity measured by their degree distribution of their signaling network.

\section{SELF-PROPELLED PARTICLES MODEL OF COLLECTIVE MOTION}

In the past two decades, a wealth of swarming models have been developed and introduced. Most of them are aimed at generating consensus behaviors [1, 5, 8, 9, 16, 17], often in the form of group alignment or polarization. In our framework, such consensus behaviors amount to swarming that refers to a circumstance in which multiple adaptive agents - be them living creatures or artificial ones - create a certain level of spatiotemporal order characterized by one or more macro-level properties.

\subsection{Base model}

Following the work by Komareji \& Bouffanais [5], we consider a collective of $\mathrm{N}$ identical and topologically interacting agents moving at the same speed. Each individual group member, at any given instant $\mathrm{t}$, is assumed to be fully characterized by a given state variable $\Psi_{\mathrm{i}}(t)$. Such a generic state variable may represent widely different characteristics depending on the nature of the group considered, but here it simply reduces to the direction of travel and the achievement of swarm consensus therefore yields an alignment of all the agents, in other words a polarized swarm. Hence, from a formal standpoint, by reaching a consensus, we mean asymptotically converging to a one-dimensional agreement in space characterized by $\Psi_{1}=\Psi_{2}=\cdots=\Psi_{\mathrm{N}}[18]$.

In the dynamical model considered, the adaptive and interacting swarming agents are modeled as self-propelled particles (SPPs) for which the biological details of the internal origin of an agent's 
thrust is considered to be irrelevant. Such SPP-based models are a good representation of collective animal behaviors [16]. Here, these SPPs are moving about a two-dimensional plane with constant speed $v_{0}$ and subject to a topological neighborhood of interaction. As mentioned previously, each agent $\mathrm{i}$ is fully characterized by its direction of travel—in other words, here $\Psi_{i}(t)=\Psi_{i}(t)$-related to the agent's velocity through $\mathbf{V i}=v_{0} \cos \theta_{\mathrm{i}} \hat{x}+v_{0} \sin \theta_{\mathrm{i}} \hat{y}$. The local synchronization protocol, based on relative states, is strictly equivalent to a local linear alignment rule which mathematically can be stated as:

$$
\dot{\theta}_{i}(t)=\frac{1}{\left|N_{i}\right|} \sum_{j \in N_{i}} w_{i j}\left(\theta_{j}(t)-\theta_{i}(t)\right),
$$

where $N_{i}$ is the set of out degree neighbors in the topological neighborhood of interaction of agent $\mathrm{i}$, with cardinal number $\left|N_{i}\right|$, and $w_{i j}$ is the binary weight of the $i-j$ communication link. Note that in some models, wij can take a more complicated form than our binary choice. Using the $k$-nearest neighbor rule to define the topological distance, we have $\left|N_{i}\right|=k$ for the case $k=7$ ) and the following dynamical equation for each individual agent $i$ in the swarm:

$$
\begin{aligned}
\dot{\theta}_{i} & =\frac{1}{k}\left[\left(\theta_{j}-\theta_{i}\right)+\left(\theta_{j+1}-\theta_{i}\right)+\cdots+\left(\theta_{j+k-1}-\theta_{i}\right)\right] \\
& =\frac{1}{k}\left(-k \theta_{i}+\theta_{j}+\theta_{j+1}+\cdots+\theta_{j+k-1}\right),
\end{aligned}
$$

where $\theta_{j}, \cdots, \theta_{j}+k-1$ are its $k$-nearest neighbors' velocity directions.

\subsection{Incorporation of noise}

Noise can generally be assumed to be random fluctuations with a normal distribution [19]. In the sequel, the background noise is considered to have a normal distribution fully characterized by its noise level, $\eta$. Specifically, the presence of noise modifies the equation governing the dynamics of agent $i$ which, using a discrete-time approach reads

$$
\theta(t+1)=\theta_{i}(t)+\frac{1}{k}\left[\left(\theta_{j}(t)-\theta_{i}(t)\right)+\cdots+\left(\theta_{j+k-1}(t)-\theta_{i}(t)\right)\right]+\eta \xi_{i}(t)
$$

where $\eta \xi_{i}(t)$ is a Gaussian white noise of magnitude $\eta$ since $\xi_{i}(t) \in[-\pi, \pi]$.

\subsection{Predator attack}

The predator is introduced as an agent that does not participate in the collective motion governed by eqn (3). Instead, it is afforded predatory capabilities associated with a motion $40 \%$ faster than swarming agents, and systematically in the direction pointing to the closest one. When the predator succeeds in catching an agent, the latter is removed from the simulation. An agent can only detect the presence of the threat when it is located at a distance smaller than a fixed radius RD; as soon as the agent detects it, an evasive maneuver is initiated with the agent moving away in the direction opposite to the predator. We have set RD to be constant throughout the simulations and independent of the established number of social links. The fleeing behavior takes precedence over the collective motion of a particular agent for as long as the predator lies inside the ball of radius RD and is associated with

$$
\vec{v}_{i}(t)=v_{0} \frac{\vec{x}_{i}(t)-\vec{x}_{i} P(t)}{\left|\vec{x}_{i}(t)-\vec{x} P(t)\right|} \text { if }\left|\vec{x}_{i}(t)-\vec{x} P(t)\right|<R_{D},
$$


where $\vec{x}$ denotes the predator's position.

The mean avoidance time shown in the next Section is obtained by computing the swarm dynamics in the presence of a single predator for 500 different runs of 5, 000 iterations each. The reason for computing several runs instead of running the calculation for longer times is that the results depend on the density of agents in the swarm, and the repeated removal of agents by the predator can cause significant changes in the density after long times.

\section{SWARM RESPONSIVENESS - PREDATOR AVOIDANCE}

In order to study the far-from-consensus dynamics of a swarm, we have simulated a predator attack as detailed in the previous Section, and measured the survival rate of agents with different levels of social interaction-corresponding to different degree distributions of the SSN, different space dimensions-two- and three-dimensional computational domains, and different levels of ambient noise $\eta$. The ubiquitous presence of noise is known to have very specific disruptive effects on the collective behavior. Intuitively, it is clear that noise corresponds to external perturbations hindering the process of self-organization.

The emergent collective avoidance maneuver is shown in Fig. 2 for two selected snapshots of a predator attack. A careful local analysis at different time instants reveals that agents outside the detection area $\left(r>R_{D}\right)$ are collectively reacting to the threat thanks to the social information transmitted through the swarm, subsequently leading to a global evasive maneuver [20]. The simulations show that the social information transfer takes place strikingly fast even though the agents themselves are considerably slower than the predator. These results are consistent with recent empirical observations of collective turns in flocks of starlings [3] and startled schools of fish [4]. Specifically, Attanasi et al. [3] have highlighted the spatially-localized origin of the triggering behavioral signal in collective turns of starlings. In addition, for a specific event involving 176 starlings, it took approximately less than $0.6 \mathrm{~s}$ for the information to flow through the whole flock, corresponding to an amazingly fast speed of information transfer of the order of $20-40 \mathrm{~m} \mathrm{~s}^{-1}$ (3 times faster than the flock itself ).

The social alignment consensus alone is sufficient for the vast majority of the agents to avoid the predator without ever detecting it. This phenomenon of augmented perceptual range in swarms due to social transfer of information is well known in flocks of birds [3] and schools of fish [21].
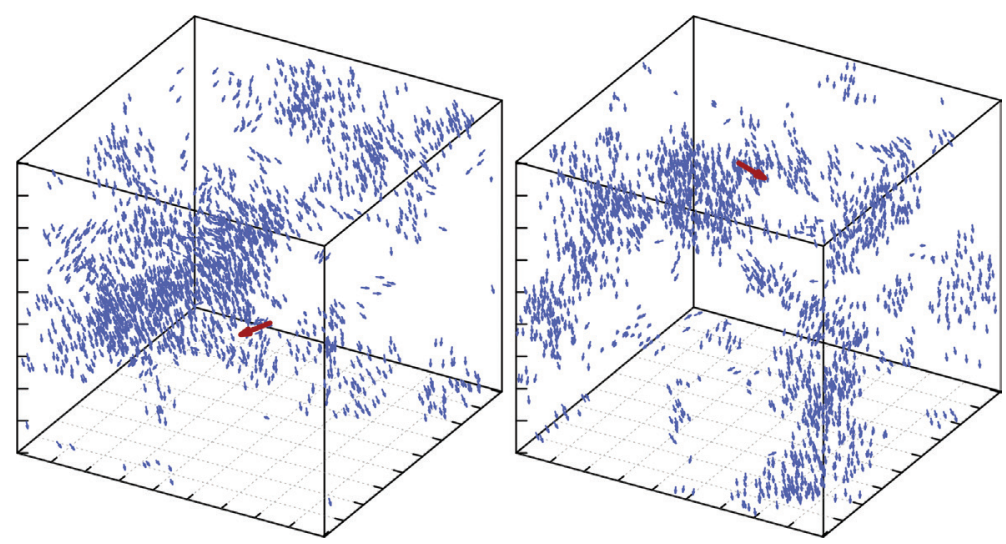

Figure 2: Snapshots of a collective evasive action induced by a predator attack at two different time instants. The $N=500 \mathrm{SPP}$ agents are in blue while the predator appears in red. 
The characteristic avoidance time for the swarm, defined as the average time elapsed between two consecutive catches by the predator, is shown in Figures 3 and 4 as a function of the number $k$ of social links between agents. Starting from a non-interacting collective $(k=0)$, the avoidance time grows with the amount of social interaction up to a maximum value about $40 \%$ larger than the non-interacting time. From that optimal point at approximately 20 neighbors, the avoidance time monotonously decreases with increasing sociality, all the way down back to the value obtained for a non-interacting collective.

While the amount of interactionquantified here as the number of neighbors or the degree distribution of the SSN - certainly has a big impact on the ability of the swarm to perform collective predator avoidance, the properties of the dynamic interaction network itself also play a fundamental role in the swarm behavior and its efficiency. One natural first step in determining how different topologies of interaction network will affect the swarm's performance is to compare the predator avoidance using the original Vicsek model [17] when the agents are embedded in a two-dimensional or a three-dimensional space (see Fig. 3). In both cases, we observe a maximum in the mean avoidance time for a finite amount of interaction. However, in the three-dimensional case the optimal number of neighbors is considerably lower than in the two-dimensional case, from $k^{*}{ }_{2 \mathrm{D}}=18$ to $k^{*}{ }_{3 \mathrm{D}}$ $=6$, consistent with the experimental observations that flocking starlings typically interact with 6 to 7 conspecifics [7]. It would be interesting to obtain similar experimental evidence for terrestrial swarms that essentially move in a two-dimensional space.

In order to be able to compare both sets of results between them, we have chosen: (i) to use a topological interaction instead of a metric one, as it reduces the number of dimensional parameters to consider, and (ii) to fix the density and speed of the agents such that the mean avoidance time in the limit for $k \rightarrow N$ is the same in both the 2D and 3D cases.

Vicsek's model [17] includes a noise term in the equations of motion to represent the agents imperfect sensing and imperfect motor capabilities. While this noise adds interesting phenomenology to the model itself (see Sec. 2.2), like a phase transition from order to disorder, it does not critically affect how the avoidance time depends on the amount of interaction. Higher noise will in

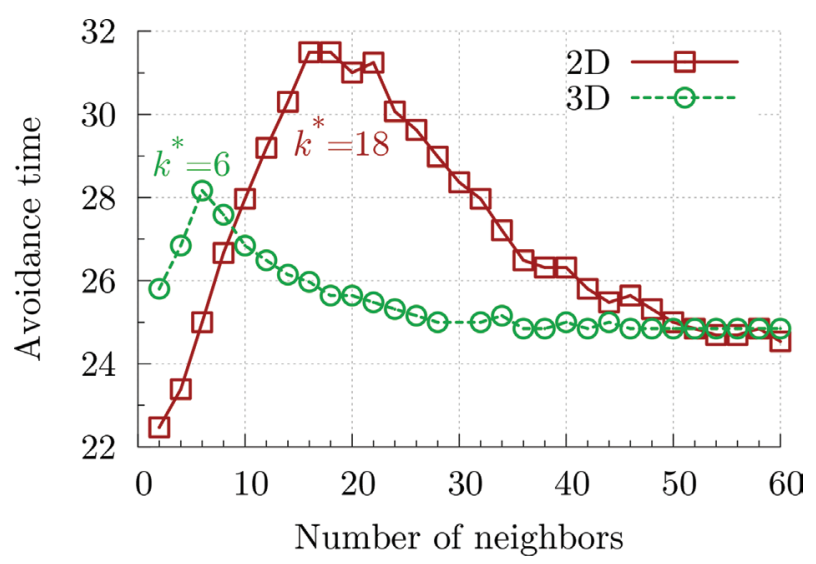

Figure 3: Efficiency in predator avoidance: Mean avoidance time for bi-dimensional and threedimensional swarms. Mean number of iterations between two consecutive predator kills (avoidance time) as a function of the number of neighbors $k$ for two-dimensional ( $\square$ ) and three-dimensional $(O)$ swarms. The number of neighbors that maximizes avoidance time in each case is shown as $k^{*}$ for a topological interaction. 


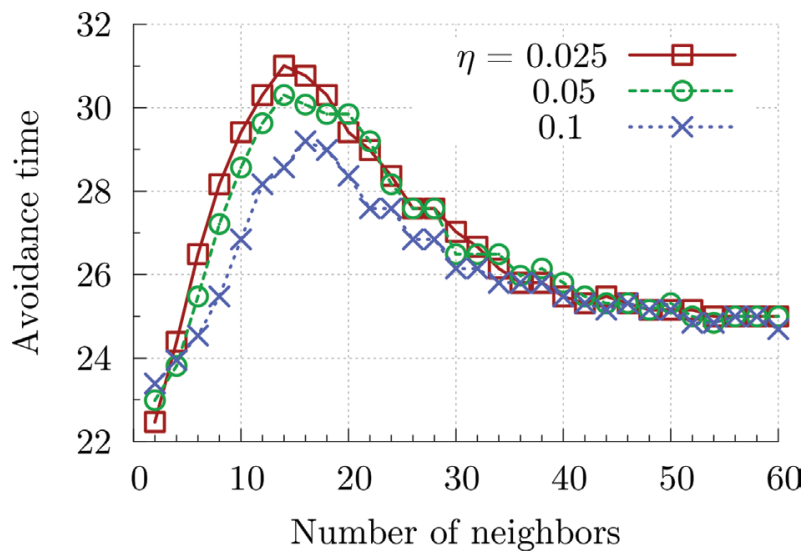

Figure 4: Influence of the ambient noise level $(\eta)$ on the effectiveness in avoiding the predator. Mean number of iterations between two consecutive predator kills (avoidance time) as a function of the number of neighbors $\mathrm{k}$ for two-dimensional swarms for a topological interaction.

general lower the peak of avoidance time but does not affect much the position of the peak itself nor change drastically the avoidance time for low and large $k$. Therefore, from the results obtained, we conclude that in this particular case the topology of the interaction network (SSN) seems to play a bigger role in determining the efficiency of the swarm in avoiding a predator than the details of the consensus protocol the swarm is following.

\section{CONCLUSION}

In our analysis, the responsiveness of swarms reveals that an excess of social activity has detrimental effects on the collective response of swarms confronted with threats and dangers. Specifically, we have shown that for a system of self-propelling agents - subjected to a consensus protocol to align their velocities - in the presence of predator. The improvement in predator avoidance with increased social activity (ascending branch in Fig. 3) reflects the expected benefits of swarming in terms of anti-predator vigilance [2, 22]. However, the existence of a maximum in the avoidance time implies that an excess of social interaction has a negative effect on the capacity of the swarm to respond to a threat associated with a dilution of behavioral information [20].

Interestingly, the avoidance time in the limit of large sociality is approximately the same as in the non-interacting swarm ( $k=0$ in Figs 3 and 4), showing that the oversaturation in the information each agent receives (i.e. the very high degree of each node of the SSN, see Fig. 1) effectively negates any benefit of social activity on the dynamical collective response. It is important noting that our results for dynamical responsiveness complement previous studies associated with global properties, such as the robustness of the interaction network [15] or the consensus speed [9]. In these studies, increasing the amount of interaction eventually yields diminishing returns - i.e. less gain per neighbor, but never an actual reduction in the property of interest. Diminishing returns can only justify the preference for a finite number of connections if the cost for establishing links between agents is significant. However, quantifying such costs is close to impossible given the complexity associated with sensory and neurological requirements in biological swarms $[9,15]$. In contrast, the present study on the dynamical responsiveness of the swarm shows an absolute reduction in swarming effectiveness when the number of neighbors is increased above a certain level. 


\section{ACKNOWLEDGMENTS}

This work was supported by a grant \#SMIG15003 from the Singapore-MIT Alliance for Research and Technology (SMART) under CENSAM (R.B.), and by a grant from the Temasek Lab (TL@ SUTD) under the STARS project (D.M.).

\section{REFERENCES}

[1] Bouffanais, R., Design and Control of Swarm Dynamics, Springer: Heidelberg, 2016. http://dx.doi.org/10.1007/978-981-287-751-2

[2] Krause, J. \& Ruxton, G.D., Living in Groups. Oxford Series in Ecology and Evolution, Oxford University Press: Oxford, U.K., 2002.

[3] Attanasi, A., Cavagna, A., Del Castello, L., Giardina, I., Grigera, T.S., Jeli'c, A., Melillo, S., Parisi, L., Pohl, O., Shen, E. \& Viale, M., Information transfer and behavioural inertia in starling flocks. Nature Physics, 10, pp. 691-696, 2014.

http://dx.doi.org/10.1038/nphys3035

[4] Herbert-Read, J.E., Buhl, J., Hu, F., Ward, A.J.W. \& Sumpter, D.J.T., Initiation and spread of escape waves within animal groups. Royal Society Open Science, 2, p. 140355, 2015.

http://dx.doi.org/10.1098/rsos.140355

[5] Komareji, M. \& Bouffanais, R., Resilience and controllability of dynamic collective behaviors. PLOS ONE, 8, p. e82578, 2013.

http://dx.doi.org/10.1371/journal.pone.0082578

[6] Holme, P. \& Saramaki, J., Temporal networks. Physics Report, 519, pp. 97-125, 2012.

http://dx.doi.org/10.1016/j.physrep.2012.03.001

[7] Ballerini, M., Cabibbo, N., Candelier, R., Cavagna, A., Cisbani, E., Giardina, I., Lecomte, V., Orlandi, A., Parisi, G., Procaccini, A., Viale, M. \& Zdravkovic, V., Interaction ruling animal collective behavior depends on topological rather than metric distance: evidence from a field study. Proceedings of the National Academy of Science USA, 105, pp. 1232-1237, 2008. http://dx.doi.org/10.1073/pnas.0711437105

[8] Komareji, M. \& Bouffanais, R., Controllability of a swarm of topologically interacting autonomous agents. International Journal Complex Systems in Science, 3, pp. 11-19, 2013.

[9] Shang, Y. \& Bouffanais, R., Influence of the number of topologically interacting neighbors on swarm dynamics. Scientific Report, 4, p. 4184, 2014. http://dx.doi.org/10.1038/srep04184

[10] Ballerini, M., Cabibbo, N., Candelier, R., Cavagna, A., Cisban, E., Gia rdina, I., Orlandi, A., Parisi, G., Procaccini, A., Viale, M. \& Zdravkovic, V., An empirical study of large, naturally occurring starling flocks: a benchmark in collective animal behaviour. Animal Behaviour, 76, pp. 201-215, 2008.

http://dx.doi.org/10.1016/j.anbehav.2008.02.004

[11] Attanasi, A., Cavagna, A., Del Castello, L., Giardina, I., Melillo, S., Parisi, L., Pohl, O., Rossaro, B., Shen, E., Silvestri, E. \& Viale, M., Finite-size scaling as a way to probe near-criticality in natural swarms. Physical Review Letters, 113, p. 238102, 2014.

http://dx.doi.org/10.1103/PhysRevLett.113.238102

[12] Gordon, D.M., Paul, R.E. \& Thorpe, K., What is the function of encounter patterns in ant colonies? Animal Behaviour, 45, pp. 1083-1100, 1993. http://dx.doi.org/10.1006/anbe.1993.1134

[13] Kawashima, H. \& Egerstedt, M., Manipulability of leader-follower networks with the rigidlink approximation. Automatica, 50, pp. 695-706, 2014.

http://dx.doi.org/10.1016/j.automatica.2013.11.041 
[14] Centola, D., The spread of behavior in an online social network experiment. Science, 329(5996), pp. 1194-1197, 2010. http://dx.doi.org/10.1126/science.1185231

[15] Young, G.F., Scardovi, L., Cavagna, A., Giardina, I. \& Leonard, N.E., Starling flock networks manage uncertainty in consensus at low cost. PLoS Computational Biology, 9(1), p. e1002894, 2013.

http://dx.doi.org/10.1371/journal.pcbi.1002894

[16] Vicsek, T. \& Zafeiris, A., Collective motion. Physics Reports, 517, pp. 71-140, 2012. http://dx.doi.org/10.1016/j.physrep.2012.03.004

[17] Vicsek, T., Cziro'k, A., Ben-Jacob, E., Cohen, I. \& Shochet, O., Novel type of phase-transition in a system of self-driven particles. Physical Review Letters, 75, pp. 1226-1229, 1995. http://dx.doi.org/10.1103/PhysRevLett.75.1226

[18] Olfati-Saber, R., Fax, J.A. \& Murray, R.M., Consensus and cooperation in networked multiagent systems. Proceedings of the IEEE, 95(1), pp. 215-233, 2007. http://dx.doi.org/10.1109/JPROC.2006.887293

[19] Dusenbery, D.B., Sensory Ecology: How Organisms Acquire and Respondto Information. W.H. Freeman and Co.: New York, 1992.

[20] Mateo, D., Kuan, Y.K. \& Bouffanais, R., Excess of social behavior reduces the capacity to respond to perturbations, 2015, arXiv:1509.08157 [nlin.AO].

[21] Sumpter, D., Buhl, J., Biro, D. \& Couzin, I., Information transfer in moving animal groups. Theory in Biosciences, 127, pp. 177-186, 2008. http://dx.doi.org/10.1007/s12064-008-0040-1

[22] Sumpter, D.J.T., Collective Animal Behavior, Princeton University Press: Princeton, NJ, 2010. http://dx.doi.org/10.1515/9781400837106 